\title{
Huiles, corps gras et produits cosmétiques
}

\author{
Alain RANCUREL \\ 3 , rue de Ouarville, 28300 Leves \\ Tél. : 0237213263. \\ <rancurel@club-internet.fr>
}

\begin{abstract}
Cosmetic and fat industries maintain close relationship of customer to supplier. Fats being considered either as excipients or as invaluable active ingredients for the cosmetic industry. It should not be forgotten that the discovery of the structure of the lipids is due to Michel Eugene Chevreul a French chemist during the $X I X^{\circ}$ century he noticed, also, the presence in small quantity of what we call today the unsaponifiable. The consumption of fat by the cosmetic industry is low as compared to the produced tonnage. It is marked by the great diversity of fats consumed either virgin or refined. They are used as excipient or as active ingredient. Fats used by cosmetic formulators must comply with the specific regulations of cosmetic industry. A promising future exists for the marketing of new fats and research of the biological activities among the minor components of lipids.
\end{abstract}

Key words: Chevreul, cosmetic, excipient, excipient, lipids corps gras conservent et que la chimie ne tue pas toujours quand elle est bien employée. En dehors de cet aspect historique, les industries de la cosmétique et des corps gras entretiennent des relations étroites de client à fournisseur, puisque de nombreux corps gras naturels sont utilisés dans les formules, comme nous le verrons au cours de ces journées. Les corps gras sont une source d'excipients de valeur, mais n'oublions pas qu'ils peuvent être aussi des principes actifs précieux pour l'industrie cosmétique et que, par ailleurs, la peau comporte un système complexe de lipides. Je vois donc les lipides à la fois comme cibles et comme vecteurs dans ce combat pour la jeunesse et la beauté que mènent les formulateurs de l'industrie cosmétique.

\section{Consommation de corps gras dans I'industrie cosmétique}

L'organisation des journées m'a demandé de vous fournir des chiffres sur l'utilisation des corps gras en cosmétique. En fait, la consommation des corps gras par cette industrie ne fait pas l'objet d'une statistique spécifique, on ne peut donc faire que des estimations; je vous invite donc à jeter quelques coups de projecteur sur quelques valeurs qui caractérisent I'usage des corps gras utilisés par la cosmétique par rapport aux usages traditionnels alimentaires.
En premier lieu, elle est marquée par la diversité, un très grand nombre de corps gras naturels essentiellement d'origine végétale sont répertoriés dans les annuaires et index de fournisseurs ; j'en ai repérés environ 200 et, si on décompte les dérivés de ces corps gras, on dépasse 1500 produits différents (encadré 1). Vous savez aussi que les contenants de l'industrie cosmétique sont de faible capacité : de quelques grammes à $200 \mathrm{~g}$ en moyenne.

Sauf exception, les formes commercialisées contiennent beaucoup d'eau, la phase grasse représente de 5 à $20 \%$ d'une émulsion et sur cette phase dite grasse les adjuvants d'émulsification sont généralement majoritaires, on peut donc dire que la consommation est très faible si on la compare à celle des usages alimentaires.

Pour une huile végétale alimentaire, I'unité de consommation annuelle est la centaine de milliers de tonnes. Pour un corps gras utilisé comme excipient en cosmétique, l'unité de compte annuelle est dans le meilleur des cas la centaine de tonnes. Pour un corps gras utilisé comme principe actif en cosmétique, l'unité de compte annuelle est la tonne, voire moins, les commandes de quelques $\mathrm{kg}$ ne sont pas rares. Nous pouvons ajouter que la durée de vie de ces matières sur le marché est très variable : de une à plusieurs années, suivant en cela les phénomènes de mode auxquels cette industrie est soumise. aujourd'hui I'insaponifiable.

En particulier, vers 1813, Chevreul retira des calculs biliaires une substance cristallisable à haut point de fusion qu'il baptisa " cholestérine » : notre cholestérol.

Chevreul fut un homme d'une exceptionnelle longévité puisqu'il est mort à 103 ans. Pour les plus curieux d'entre vous, je vous invite à consulter le numéro de La Recherche de ce mois de mars où figurent quelques photos de Chevreul prise par Nadar dans sa centième année. Si je puis me permettre, voilà qui prouve que les 


\begin{tabular}{|c|}
\hline 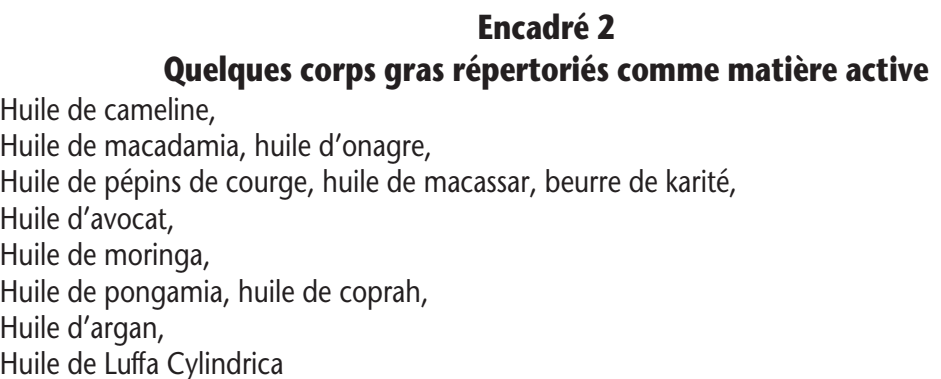 \\
\hline
\end{tabular}

Il est également difficile de donner un ordre de grandeur des prix. Cependant, on peut retenir une fourchette de 2 à 100 euros.

\section{Usages des corps gras dans I'industrie cosmétique}

Nous devons préciser immédiatement que I'utilisation d'une substance dans ces industries peut se faire sous deux formes: excipient ou principe actif.

Nous serons amené à préciser les règles d'emploi dans l'un et l'autre cas.

\section{Excipient}

Les corps gras sont utilisés soit à l'état naturel, soit bruts, soit raffinés ou sous forme de dérivés tensioactifs, ou autre. L'encadré 1 présente quelque-uns de ces dérivés.

Quelles sont les formules concernées?

- les formules sans eau : rouge à lèvres, compacts, etc. ;

- les formules émulsionnées ils font alors partie de la phase grasse d'émulsion eau dans huile, huile dans eau ou d'émulsion multiples.

L'excipient est certes un vecteur mais « intelligent ", il doit en effet le plus souvent contribuer à solubiliser le "principe actif » (nous reviendrons sur ce terme); il contribue de façon parfois décisive aux qualités organoleptiques: couleur, odeur et toucher du produit fini.

II doit donc être neutre de ce point de vue, c'est-à-dire qu'il ne doit pas présenter de réaction fâcheuse avec les autres constituants de la formule, il ne doit pas avoir de toxicité propre, il doit être intrinsèquement stable. Tous ces points seront développés au cours de ces journées.

\section{Matière active}

Je me répète, mais il faut insister : la variété des substances tirées des corps gras et utilisées en tant que matières actives ou revendiquant une activité est très grande (encadré 2).

On trouve de nombreuses huiles végétales. Une bibliographie récente $\mathrm{m}^{\prime} \mathrm{a}$ permis de

II doit ne pas contenir de polluants indésirables: aflatoxine, hydrocarbures aromatiques polycycliques, résidus de pesticides, OGM en partant des plus anciens vers les plus récents. Tous ces aspects vont êtres développés et je l'espère discutés au cours de notre première journée.

\section{Contraintes réglementaires en cosmétique}

Rappelons à cette occasion la définition d'un produit cosmétique selon la Communauté européenne et reprise dans le code de la santé publique (encadré 3).

Cette définition pose le problème des principes actifs utilisés en cosmétique puisque d'après ce texte un constituant du produit ne doit pas pénétrer le corps humain. Où se trouve la limite entre le médicament et le cosmétique?

Nous aurons certainement à en débattre au cours des conférences de notre deuxième journée.

\section{Le futur, les innovations, les attentes}

Voici le moment difficile : prendre sa boule de cristal et faire des projections qui seront démenties quand on les relira dans quelques années (heureusement que je ne me fais pas d'illusion sur une relecture de ma prose).

Si I'on reprend ma division précédente, on peut dire que les développements peuvent intervenir dans trois domaines : I'excipient, le principe actif et la formule.

le pense que l'industrie cosmétique va continuer d'évoluer vers de nouveaux modes de formulation grâce aux progrès des agents de surface et aux adjuvants de formulation.

On peut envisager des développements vers les émulsions multiples, les microémulsions, la microencapsulation, I'utilisation des molécules cages comme les cyclodextrines dans le cas de molécules fragiles.

Par contre, le développement de formes utilisables pour une cosmétique par voie orale me paraît limité par la réglementation, nous pourrons en débattre.

En ce qui concerne le principe actif, I'industrie des corps gras doit continuer à proposer des produits nouveaux issus de plantes rares et être

\section{Encadré 3}

\section{Définition d'un produit cosmétique selon la Communauté européenne}

On entend par produit cosmétique « toute substance ou préparation destinée à être mise en contact avec les diverses parties superficielles du corps humain, notamment l'épiderme, les systèmes pileux et capillaires, les ongles, les lèvres et les organes génitaux externes, ou avec les dents et les muqueuse buccales, en vue exclusivement, ou principalement, de les nettoyer, de les parfumer d'en modifier l'aspect, de les protéger, de les maintenir en bon état, ou de corriger les odeurs corporelles. » 
capable de produire des corps gras de haute qualité en petit tonnage.

Les progrès des connaissances en biologie et en physiologie devraient continuer à permettre la mise en évidence des propriétés de ces nouveaux corps gras ou de découvrir des vertus à des substances déjà connues. C'est ce que j'ai pu constater à la lecture de brevets récents concernant l'huile d'avocat.

\section{Conclusion}

Nous aurions pu donner comme titre à ces journées: «Tout ce que l'industrie des corps gras a toujours voulu savoir sur la cosmétique sans avoir jamais osé le demander et tout ce que l'industrie de la cosmétique a toujours voulu savoir sur les corps gras (pardon, les lipides) sans avoir jamais osé le demander ».

Alors osons. 